\title{
DESEMPEÑO DE ESTUDIANTES DE INGENIERÍA CIVIL DESDE LA PERSPECTIVA DE LOS EMPLEADORES, PERIODO 2014-2016
}

\section{PERFORMANCE OF CIVIL ENGINEERING STUDENTS THE PERSPECTIVE OF EMPLOYERS, PERIOD 2014-2016}

(Recibido/received: 20-Abril-2020; aceptado/accepted: 6-Junio-2020)

RESUMEN: El artículo refiere a la evaluación de la percepción de los empleadores en el desempeño laboral de los estudiantes de la carrera de ingeniería civil, de la Universidad Nacional de Ingeniería, Sede Regional del Norte, en la ciudad de Estelí, Nicaragua, en el periodo 20142016, durante la realización de prácticas pre - profesionales o pasantías en instituciones regionales estatales, como privadas. Se realizó análisis estadístico descriptivo de los datos, análisis de varianza multivariado, así como una prueba "t" que indicó que no existe una diferencia significativa en la evaluación del desempeño estudiantil tanto del sector privado como estatal para las 66 organizaciones de las que se tiene registro de datos. Se generó un gráfico BIPLOT, con el cual es posible inferir que en el sector privado se evalúan mejor los aspectos relacionados a la capacidad de integrarse al trabajo en equipo, la iniciativa y capacidad para analizar, así como el cuido y resguardo de los bienes. La percepción de los empleadores del desempeño de los estudiantes es excelente, marcando una pauta para continuar con las prácticas o pasantías para fortalecer el vínculo con el entorno, consolidar la experiencia de estudiantes y poder así sumar a la formación integral de los graduados en esta casa de estudios.

PALABRAS CLAVE: Competencias de graduados, formación integral, educación superior, gestión de la educación.

ABSTRACT: The article refers to the evaluation of employers' perception of the job performance of civil engineering students from the National University of Engineering, Regional Campus of the North, in the city of Estelí, Nicaragua, in the period 2014-2016, during the completion of pre-

\footnotetext{
1 Universidad Nacional de Ingeniería Estelí, Nicaragua. Correo: Sergio.Navarro@norte.uni.edu.ni, Orcid: https://orcid.org/0000-0002-7001-5860

2 Universidad Nacional de Ingeniería Estelí, Nicaragua. Correo: Sandra.Blandon@norte.uni.edu.ni, Orcid: https://orcid.org/0000-0002-5450-7507
} 
professional internships in governmental and private regional institutions. It was performed a descriptive statistical analysis of the data, a multivariate variance analysis as well as a "t" test, which indicated that there is no significant difference in the evaluation of the student performance of both the private and governmental sectors for the 66 organizations with data registered. A BIPLOT graph was generated, which it possible to infer that in the private sector the aspects related to the ability to integration to the teamwork, the initiative and ability to analyze, as well as the care and protection of assets, are better evaluated. The perception of employers of student performance is excellent, setting a pattern to continue with internships or training to strengthen the bond with the environment, consolidate the student experience and thus be able to add to the comprehensive training of those graduated in this house of studies.

KEYWORDS: Graduates competencies, integral training, higher education, educational management

\section{INTRODUCCIÓN}

Como refieren Ramírez y García (2010) las instituciones de educación superior promueven alianzas estratégicas con las empresas, organismos e instituciones de su entorno que le permitan establecer como un referente en la producción de resultados que respondan a demandas del sector productivo, logrando así contribuir al fortalecimiento de su misión y visión en cuanto a desarrollo de las ciencias y de las artes, que permitan la actualización constante en sus procesos internos. Refieren que:

La construcción de puentes entre Gobierno, Universidad y Empresas es vital para la utilidad del capital humano en el desarrollo, como núcleo que es de las capacidades científicas y tecnológicas, las cuales en el país se encuentran, por una parte, focalizadas en las unidades regionales ya mencionadas, y por otra parte, diseminadas por su falta de relación y coherencia con los sectores productivos. Las limitaciones existentes tienden a superarse mediante diseños organizacionales más horizontales, descentralizados y flexibles que permitan a la universidad responder ágilmente y con propiedad a las demandas de innovación (Ramírez y García, 2010, p. 128).

Por su parte Castillo, Álvarez y Torres (2013) indican que la vinculación de la universidad con el entorno no es un tema nuevo, continúan su planteamiento indicando que:

Se ha ido perfeccionando en función de su misión, en la medida que el desarrollo científico técnico avanza, este problema debe ir transformándose. En la actualidad urge una mayor interacción de manera tal que se favorezcan los procesos de gestión y formación, y que esto a su vez conlleve a un mayor beneficio de la sociedad. Se percibe falta de claridad y de estrategias para el impacto de ese vínculo en el desarrollo de cada uno de los factores participantes y de la sociedad en general (p. 4).

El Higo Revista Científica / Volumen 10. No. 01, pp. 53-62 / Junio 2020 
La capacidad de las universidades para afrontar los grandes cambios de la actualidad y aprovechar las oportunidades que presentan las nuevas circunstancias de su entorno, se incrementará en la medida en que dispongan de una infraestructura adecuada y recursos suficientes para subsidiar su desarrollo institucional y poder cumplir con el compromiso social que las caracteriza y así, ser reconocidas por la comunidad a la que sirven (Alcántar y Arcos, 2009, p. 4).

Siempre se ha creído que una inclusión de los empleadores para valorar la calidad de la enseñanza reflejada en las habilidades y desempeño de los graduados será de alta significación; de hecho la evaluación de parte de los empleadores y los resultados podrán servir de base para la generación de ideas y acciones prospectivas para ser incorporadas en los planes de estudio que permitan dar respuesta oportuna, activa y pertinente a los demandas del entorno en correspondencia con la razón de ser de las instituciones educativas (Chung y Yet, 2009).

Una manera de cumplir los requerimientos del entorno por parte de las universidades es por medio de la formación de profesionales más preparados con un alto sentido de corresponsabilidad social, por esto, es prioritario contar con los mecanismos necesarios, favorecedores de los procesos de vinculación para la generación de los beneficios de los participantes.

Selvadurai, Choy y Maros (2012) plantean algunas recomendaciones de como cumplir los requerimientos del entorno. Expresan que:

En la actualidad, es urgente una mayor interacción de las universidades con el entorno de tal manera que se favorezcan los procesos de gestión y formación, y que esto a su vez conlleve a un mayor beneficio de la sociedad. Para ello es necesario tomar en cuenta la demanda (relación con el entorno) y perspectiva de los empleadores quienes son los que evaluarán el desempeño de los formados en la universidad, considerando que estos en el contexto actual requieren habilidades y atributos tanto de carácter personal como intelectual, tales como la capacidad de comunicación y gestionar información, el trabajo en equipo con interacción social, capacidad para resolver problemas con alta capacidad de autogestión así como el liderazgo, mismos que requieren participar en intercambios de experiencias con las empresas, motivar el autoaprendizaje permanente, la lectura y cultura investigativa (pp. 298-301).

La Universidad Nacional de Ingeniería (UNI), en Nicaragua, es una institución de referencia regional en la formación de ingenieros y arquitectos desde hace más de treinta y cinco años. Con visión estratégica, en el 2004 se fundó la Sede Regional del Norte, en la ciudad de Estelí, en la cual se ofertan cuatro carreras de ingeniería, siendo la más demandada Ingeniería Civil, misma que representa actualmente más del $60 \%$ de la población estudiantil. En cumplimiento con su misión y visión, la universidad asume el compromiso constante de la formación integral de egresados que den respuesta, de manera eficaz y eficiente a los problemas y necesidades del entorno en que se desempeñan, contribuyendo al desarrollo del país.

El Higo Revista Científica / Volumen 10. No. 01, pp. 53-62 / Junio 2020 
En el año 2016 se realizó una mejora a los programas del plan 97 de la carrera de ingeniería civil como parte de los procesos de autoevaluación y mejora, sin embargo en esta no se integró al programa de la carrera la realización de prácticas pre profesionales o pasantías. Particularmente en la Sede Regional se han desarrollado mecanismos internos que permiten a los estudiantes de tercero y cuarto año realizar prácticas pre- profesionales en las instituciones, tanto a nivel local como regional.

En este artículo se hace un análisis cuantitativo y cualitativo del desempeño de estudiantes de ingeniería civil desde la perspectiva de los empleadores, acorde a la información disponible en el periodo 2014-2016.

\section{METODOLOGÍA}

De acuerdo al propósito y valor el estudio es descriptivo y correlacional Hernández, Fernández y Baptista (2014, pp. 97-98). De acuerdo, al tiempo de ocurrencia de los hechos y registro de la información, el estudio tiene un diseño no experimental transaccional (Hernández, et al,. pp. 154155). Para el procesamiento de los datos y obtención de estadísticas descriptivas así como gráficos se utilizó el software de InfoStat (Balzarini, Tablada, Casanoves, Di Rienzo y Robledo, 2008, p.13).

Se analizó la perspectiva de los empleadores retomando una evaluación de 46 empleadores de carácter privado (Incluyendo contratistas de la región) así como 22 de carácter estatal sistematizadas en 185 casos de estudio, las cuales cumplen las condiciones de validez interna e incluye a los estudiantes activos que cursaban los terceros y cuartos años de su pensum académico (ver tabla 1 y tabla 2). Se empleó el análisis de la varianza multivariado (MANOVA), que permitió la inferencia de efectos en los factores para el modelo de análisis propuesto. Se aplicó una prueba t, para probar la hipótesis, asumiendo que son muestras independientes. Se construyó un gráfico BIPLOT para mostrar las observaciones para las variables estudiadas y analizar sus relaciones (Balzarini et al., 2008, pp. 189-204).

Tabla 1. Organizaciones estatales y no gubernamentales

\begin{tabular}{cc}
\hline Descripción de organización & Institución \\
\hline Alcaldía Municipal & Achuapa \\
Alcaldía Municipal & Ciudad Darío \\
Alcaldía Municipal & Estelí \\
Alcaldía Municipal & Nueva Segovia \\
Alcaldía Municipal & San Lucas, Madriz \\
Alcaldía Municipal & Achuapa \\
Alcaldía Municipal & San Isidro \\
Alcaldía Municipal & Dipilto \\
Alcaldía Municipal & La Trinidad \\
Alcaldía Municipal & Jícaro \\
Alcaldía Municipal & Jinotega \\
\hline
\end{tabular}

El Higo Revista Científica / Volumen 10. No. 01, pp. 53-62 / Junio 2020 


\begin{tabular}{rc}
\hline Alcaldía Municipal & La Trinidad \\
Alcaldía Municipal & Ocotal \\
Alcaldía Municipal & San Lucas \\
Alcaldía Municipal & Sébaco \\
Alcaldía Municipal & La Dalia \\
ENACAL & Estelí \\
Universidad Nacional de Ingeniería - UNI & Estelí \\
Fondo de Mantenimiento Vial - FOMAV & Managua \\
Ministerio de Educación - MINED & Estelí \\
Ministerio de Transporte e infraestructura - MTI & Managua \\
Nuevo FISE & Jinotega \\
\hline
\end{tabular}

Tabla 2. Organizaciones privadas

\begin{tabular}{ll}
\hline \multicolumn{1}{c}{ Descripción de organización } & \multicolumn{1}{c}{ Institución } \\
\hline Arje Proyect 3D & Creación e ingeniería civil \\
ARQUINGTOP & D.A.M.L construcción verticales y horizontales \\
Bayardo Jesús Gómez & Diseños creativos S.A \\
BBB & Diseños presupuestos 3 desing arquitectura \\
Contratista & EDICO - Estudios y diseños Ing consultores. \\
COICSA & FERPLO \\
Ladrillera San Pablo S.A & Hego-renta de equipos \\
Construcción López Lorente & HFTL todo en diseño y construcción \\
Construcción verticales y horizontales & Hotel ecológico bella vista \\
Construcciones Alvarado & I. CONST \\
Construcciones Pérez & Matute construcciones \\
Construcciones verticales y horizontales & Oficina de arquitectura \\
Construcciones y diseños Cárdoza Méndoza & Proyectos y diseños creativos SA. (PRODICSA) \\
Constructora de obras de Ingeniería civil S.A & INNOVA Estelí \\
Constructora Edgar Delgado Blandón & Rizo Pineda Consultorías y Asesorías \\
Constructora Hernández & IELEMNIC \\
Constructora López Lorente & Servicio de Construcción Javier Otero \\
Constructora Mairena & Servicios Eléctricos Calderón \\
Constructora Mario González & Servicios Múltiples de Construcción \\
Constructora Pablo García H. & SINAR S.A. \\
Constructora Vílchez & TECS. Topografía especializada \\
Constructora Mario González & Transporte y maquinarias J.M.O \\
COTESA & Vegas NICSA \\
\hline
\end{tabular}




\section{RESULTADOS Y DISCUSIÓN}

Para la valoración de las prácticas se elaboró instrumento, mismo que fue recepcionado y resguardado por el coordinador de carrera de la Sede Universitaria. En los aspectos relacionados a la eficacia y eficiencia reflejada por los estudiantes se incorporó la motivación (Interés por aprender), la presentación personal (vestimenta acorde a perfil de trabajo), la puntualidad, asistencia y cumplimiento de horarios en su ubicación, las relaciones interpersonales establecida, el cumplimiento de tareas y objetivos asignados, actitudes del cuido y resguardo de los bienes así como material asignado, su capacidad para comunicarse y adaptarse así como su capacidad para integrarse y trabajar en equipos multidisciplinares, mismas que concuerdan con Chung y Yet (2009) y Selvadurai, et al (2012) quienes listan de habilidades claves que vinculan a las evaluadas en estos resultados. De igual manera se evaluó en perspectiva la iniciativa y capacidad de analizar y resolver problemas incluyendo el nivel de aprovechamiento del aprendizaje en el centro de práctica, cerrando con una valoración global cualitativa de cumplimiento de actividades, actitud y desempeño en una escala cualitativa de cero a cien.

Se realizó un análisis de la varianza multivariado (Tabla 3), empleando como contraste la prueba de Hotelling considerando un nivel de significación con p-valor de 0.05 , para evaluar las perspectivas de los empleadores del sector público como privado; no encontrándose diferencia significativa en la evaluación entre estos dos grupos. De igual manera se realizó una prueba de inferencia basada en dos muestras (Tabla 4) para cada variable que confirmó la no existencia de diferencias estadísticas significativas en la evaluación de los estudiantes practicantes, tanto cualitativa como cuantitativa desde la perspectiva de empleadores en análisis.

Tabla 3. Análisis de la Varianza Multivariado (ANAVAM)

\begin{tabular}{|llllll|}
\hline Resultados de & Análisis de la Varianza (Lawley & -Hotelling) & \\
\hline F.V. & Estadístico & $\mathrm{F}$ & $\mathrm{gl}($ (num) & $\mathrm{gl}(\mathrm{den})$ & $\mathrm{p}$ \\
Tipo de institución & 0.06 & 0.87 & 11 & 173 & 0.5734 \\
\hline
\end{tabular}

Nota: Elaboración propia a partir de datos generados con InfoStat

Tabla 4. Prueba " $\mathrm{t}$ " para muestras independientes por variable

\begin{tabular}{|lll|}
\hline Variable & “t” & $\begin{array}{l}\text { P- } \\
\text { Valor }\end{array}$ \\
\hline Interés por aprender & 0.84 & 0.40 \\
\hline Presentación personal & 0.92 & 0.36 \\
\hline Puntualidad y cumplimiento de actividades & 1.38 & 0.17 \\
\hline Relaciones interpersonales & -1.28 & 0.20 \\
\hline Cumplimiento de tareas & -0.80 & 0.42 \\
\hline Cuido de bienes & 1.00 & 0.32 \\
\hline Capacidad de comunicarse y adaptarse & 0.00 & 0.99 \\
\hline Capacidad de trabajo en equipo & -0.10 & 0.92 \\
\hline
\end{tabular}

El Higo Revista Científica / Volumen 10. No. 01, pp. 53-62 / Junio 2020 


\begin{tabular}{|lll|}
\hline Capacidad de resolver problemas & -0.16 & 0.87 \\
\hline Aprovechamiento de las prácticas & 0.50 & 0.62 \\
\hline Evaluación global del desempeño & 0.97 & 0.33 \\
\hline
\end{tabular}

Aunque no se encontraron diferencias significativas entre la evaluación y los empleadores se realizó un análisis multivariado con un análisis de componentes principales consolidado en un BIPLOT (ver figura 1) que infiere una tendencia en aspectos evaluativas de los empleadores de empresa privada tales como la capacidad de integrarse en equipo, la iniciativa y capacidad para analizar así como el cuido y resguardo de los bienes. El trabajo de Selvadurai, et al. (2012) menciona habilidades genéricas de los alumnos egresados generadas desde el contexto universitario, la inserción laboral (las prácticas o pasantías) así como el empleo mismo, las cuales coinciden con las evaluadas por los empleadores de la región tales como el trabajo en equipo, capacidad de resolver problemas, capacidad de comunicarse y adaptarse. Cabe indicar que en todos los aspectos evaluados los estudiantes (ver figura 2) que realizan prácticas son valorados en rangos de evaluación cuantitativa que alcanza los 94.6 puntos ponderados.

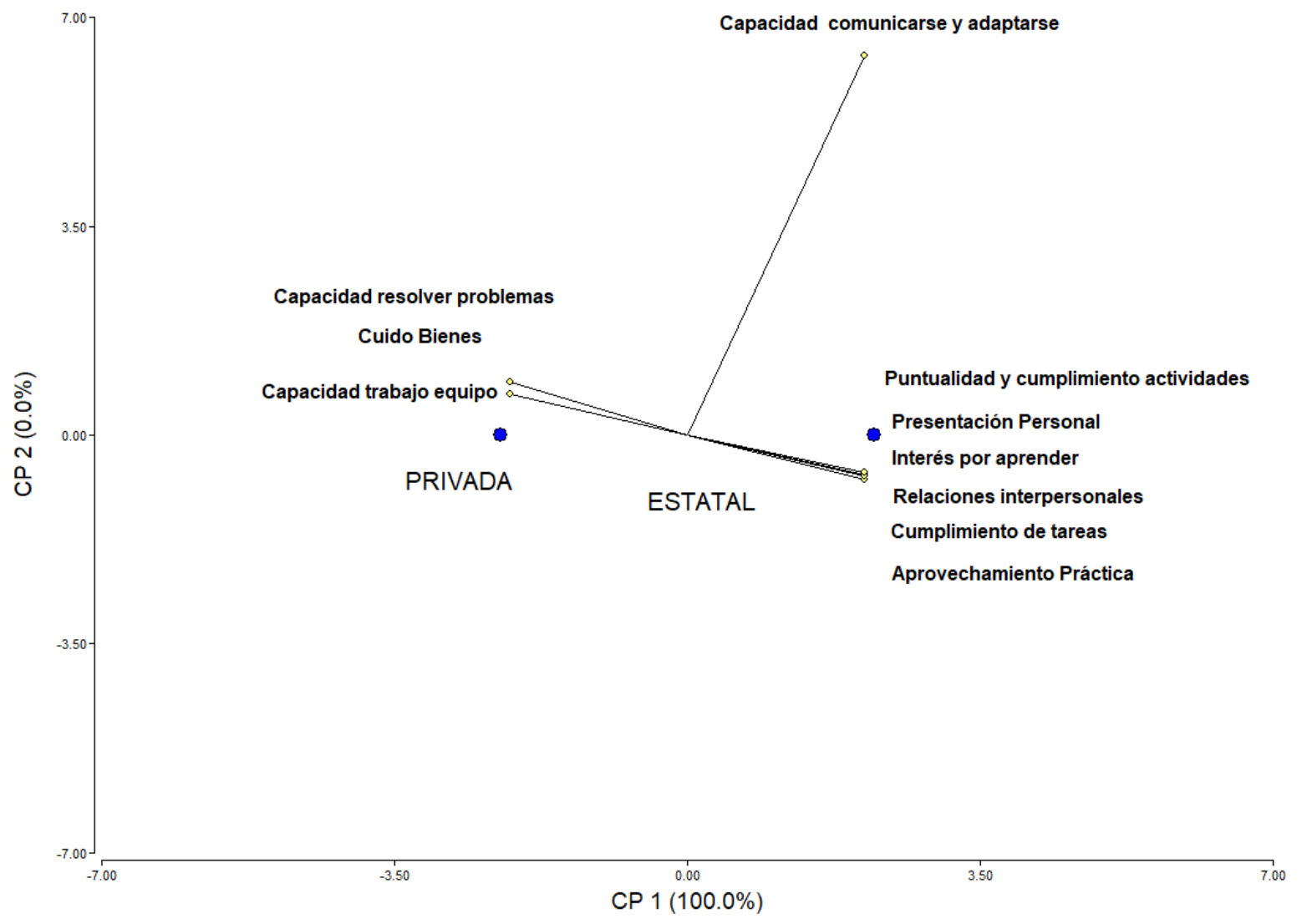

Figura 1. BIPLOT. Tendencia en aspectos evaluados de los empleadores de empresa privada y estatal.

El Higo Revista Científica / Volumen 10. No. 01, pp. 53-62 / Junio 2020 
Cuido y resguardo de los bienes así como material asignado

Interés por aprender

Cumplimiento de tareas y objetivos asignados

Presentación personal

Aprovechamiento del aprendizaje en el centro de práctica

Valoración global cualitativa de cumplimiento de actividades, actitud y desempeño

Capacidad para integrarse y trabajar en equipos multidisciplinares

Capacidad para comunicarse y adaptarse

Relaciones interpersonales

Puntualidad, asistencia y cumplimiento de horarios

Iniciativa y Capacidad de analizar y resolver problemas

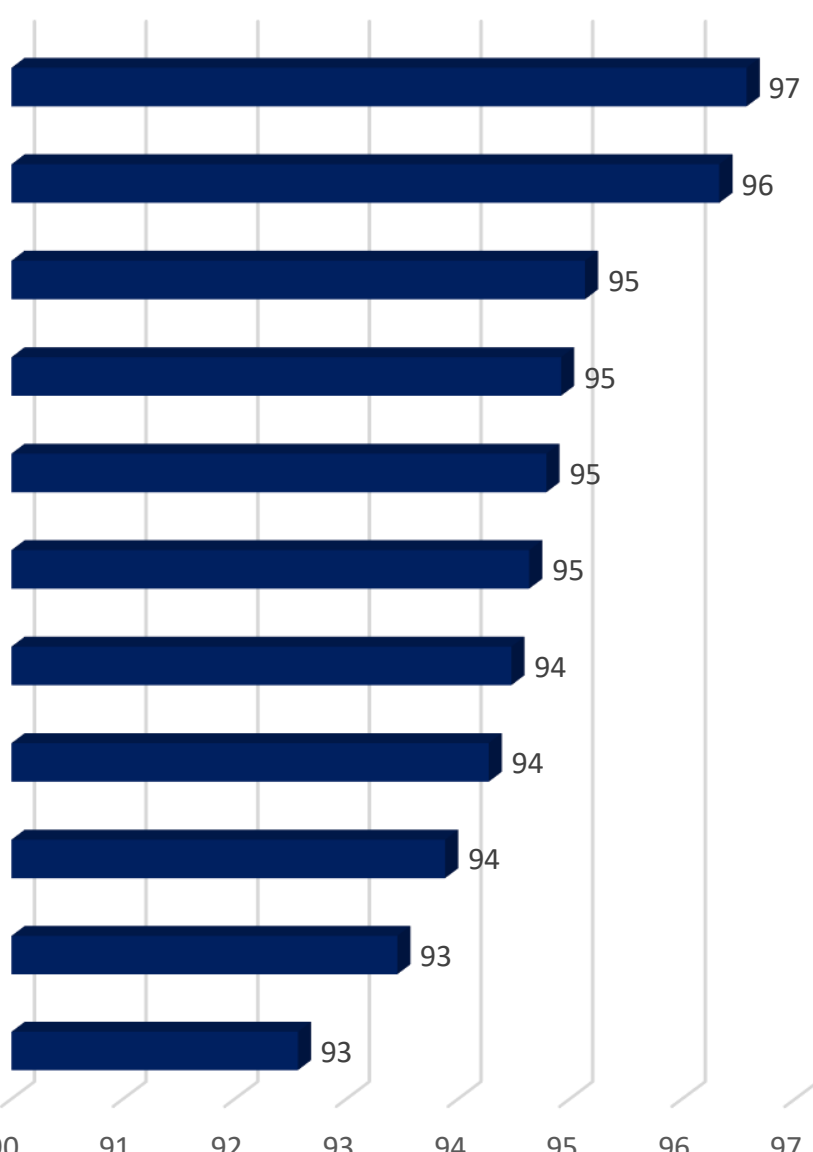

Figura 2. Perspectiva evaluativa del desempeño de estudiantes practicantes de ingeniería civil.

\section{CONCLUSIONES}

La percepción del desempeño de los estudiantes de ingeniería civil de la Universidad Nacional de Ingeniería, Sede Regional en Estelí, que realizaron prácticas o pasantías, tanto de los empleadores de carácter privado como estatal, con evaluaciones en escala cuantitativa de cero a cien, supera los noventa y cuatro puntos en promedio, no evidenciando diferencias significativas en su evaluación.

Los empleadores de la región norte de Nicaragua, incluidos en este análisis, tienen una percepción muy positiva del desempeño de los estudiantes formados en la Universidad Nacional de Ingeniería, no obstante, es necesario que se continúen sistematizando estas experiencias, que abonen a la identificación de oportunidades para la mejora de los procesos internos que den

El Higo Revista Científica / Volumen 10. No. 01, pp. 53-62 / Junio 2020 
respuesta a la demanda del entorno que favorezcan la inserción y exitoso desempeño laboral de los estudiantes de la carrera de ingeniería civil.

Se sugiere realizar una incorporación al pensum académico, que fomente las prácticas o pasantías como una estrategia para la formación integral de los formados en esta institución, en correspondencia con la misión y visión universitaria. Es necesario el fortalecimiento de esfuerzos institucionales para incluir la realización de prácticas pre - profesionales transversales al desarrollo de la carrera de ingeniería civil, mismo que estén orientados al incremento del impacto positivo del capital educativo de los graduados en el mercado de trabajo.

Se resalta que son las pasantías una oportunidad en la que el estudiante pueda poner en práctica lo aprendido y desde luego tomar experiencia entorno a la práctica laboral; representando también una apertura de la universidad hacia el contexto social, una vinculación que permite la participación en los procesos de formación profesional de los estudiantes antes de su egreso, que aumenta las posibilidades de empleabilidad y, sobre todo, su experiencia pre profesional.

\section{REFERENCIAS}

Alcántar, V. y Arcos, J. (2009). La vinculación como factor de imagen y posicionamiento de la Universidad Autónoma de Baja California, México, en su entorno social y productivo. REDIE, Revista Electrónica de Investigación Educativa, 11(1), 1-13.

Balzarini, M., Tablada, E., Casanoves, F., Di Rienzo, J. y Robledo, C. (2008). Manual del Usuario. Córdoba: Editorial Brujas.

Castillo, J., Álvarez, N. y Torres, A. (2013). La vinculación de las universidades mexicanas con el entorno: expresión de responsabilidad social. Transformación, 9(2), 1-13.

Chung, W. y Yet, L. (2009). Perception Differential between Employers and Undergraduates on the Importance of Employability Skills. International Education Studies, 2(2), 95-105.

Hernández, R., Fernández, C. y Baptista, M. (2014). Metodología de la investigación (6 ed.). Mexico: Mc Graw Hill.

Ramírez, M. y García, M. (2010). La Alianza Universidad-Empresa-Estado: una estrategia para promover innovación. Revista EAN, 68, 112-133.

Selvadurai, S., Choy, E. y Maros, M. (2012). Generic Skills of Prospective Graduates from the Employers' Perspectives. Asian Social Science, 8(12), 295-303. 


\section{SEMBLANZA DE LOS AUTORES}
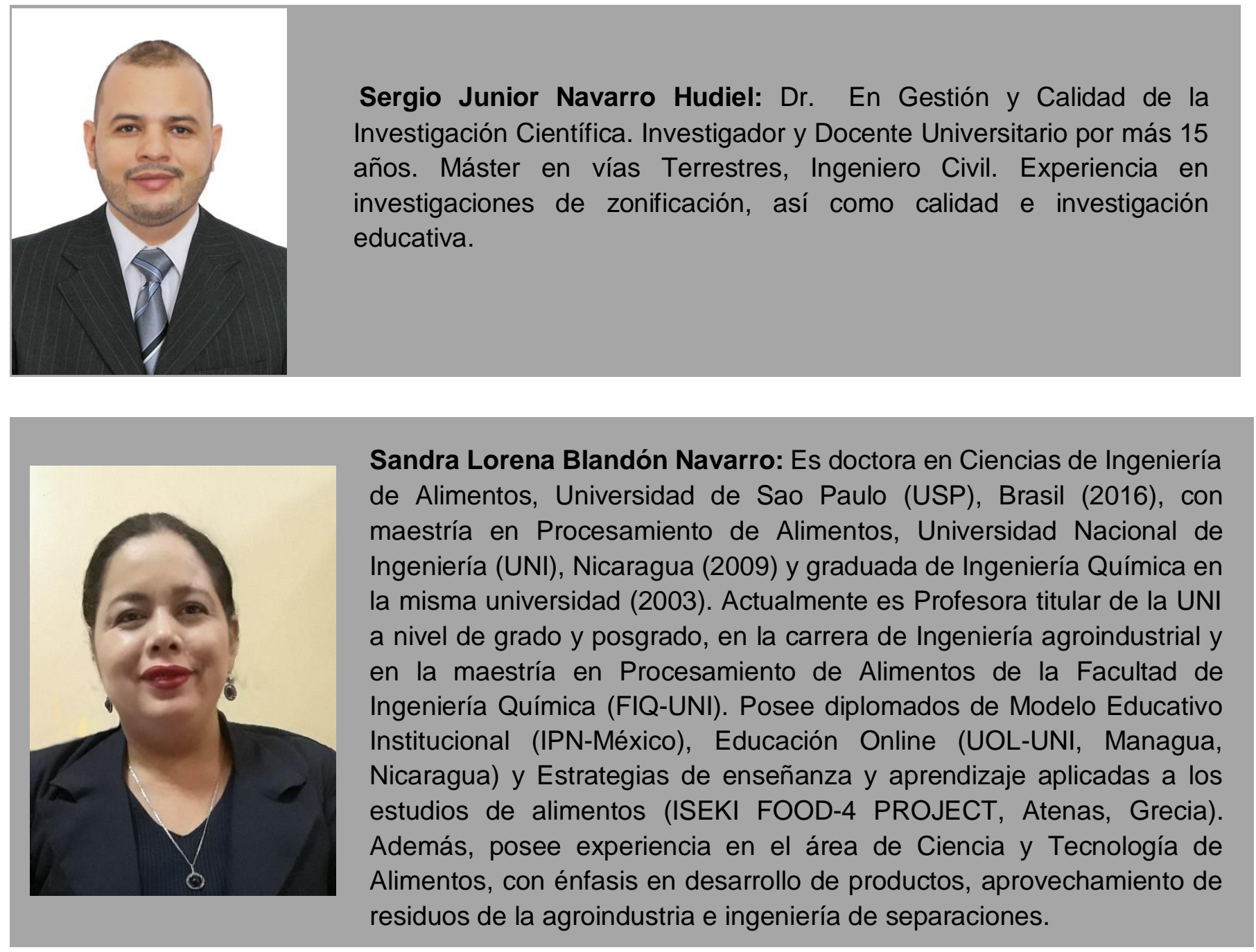

El Higo Revista Científica / Volumen 10. No. 01, pp. 53-62 / Junio 2020 